\title{
Rapid gene cloning in wheat
}

\begin{abstract}
The identification of wheat and barley genes controlling important agronomic traits using positional cloning has traditionally been a challenging and time-consuming procedure. This is due to the enormous genome size and high repeat content from transposable elements (TEs). Low marker density, suppressed recombination, and the high cost of generating a physical contig across a genetically defined map interval have further restricted the application of positional approximation. Over the past decade, the cost of DNA sequencing has significantly dropped, as has our ability to computationally analyze large quantities of DNA sequence data. This has enabled researchers to exploit next-generation sequencing (NGS) technologies more routinely to accelerate the gene cloning process. In this chapter, we discuss several newly emerging cloning methods that combine NGS technologies with recent advances in molecular genomics to overcome previous limitations of gene cloning in wheat and barley.
\end{abstract}

Keyword: Trends; Traffic pollutant; Localized; Interpolation 